\title{
Efeitos da fisioterapia aquática em prematuros internados na unidade de Terapia
}

\section{Intensiva Neonatal}

\author{
Effects of aquatic physiotherapy in hospitalized premature in the Neonatal Intensive Therapy Unit \\ Efectos de la fisioterapia acuática sobre prematuras admitidas a Unidad de Cuidado Intensivo
}

Neonatal

Recebido: 14/07/2021 | Revisado: 03/08/2021 | Aceito: 23/08/2021 | Publicado: 25/08/2021

\author{
Daniela Cristina Rambo \\ ORCID: https://orcid.org/0000-0003-3876-4674 \\ Universidade Franciscana, Brasil \\ E-mail: danielarambo@hotmail.com \\ Nadiesca Taisa Filippin \\ ORCID: https://orcid.org/0000-0003-3140-2486 \\ Universidade Franciscana, Brasil \\ E-mail: nadifilippin@ufn.edu.br \\ Clândio Timm Marques \\ ORCID: https://orcid.org/0000-0002-9984-0100 \\ Universidade Franciscana, Brasil \\ E-mail:clandiomarques@gmail.com
}

\begin{abstract}
Resumo
Objetivo: Verificar os efeitos da fisioterapia aquática em prematuros internados na Unidade de Terapia Intensiva Neonatal. Método: Trata-se de um ensaio clínico não randomizado. A amostra foi composta por 15 recém-nascidos prematuros. Os dados pessoais e clínicos dos prematuros foram coletados do prontuário. Eles foram avaliados em dois dias consecutivos: no primeiro dia, foi realizada a avaliação das variáveis do estudo (frequência cardíaca e respiratória, saturação periférica, pressão arterial, dor e sono); logo após, foi feita a punção sanguínea para coleta de exames de rotina e, por fim, foram reavaliadas as variáveis. No segundo dia, foram repetidos os procedimentos do primeiro dia; no entanto, após a punção sanguínea foi realizada fisioterapia aquática. Resultados: Foi possível observar que as variáveis frequência cardíaca $(\mathrm{p}<0,01)$ e sono $(\mathrm{p}<0,01)$ mostraram diferenças significativas entre pré e pós-intervenção. As variáveis saturação, frequência respiratória e dor mostraram diferenças significativas entre pré e pós-procedimento do primeiro dia, mas não entre pré e pós intervenção. No entanto, foi possível observar diferenças entre a avaliação pós do primeiro dia e pós do segundo dia. Conclusão: Os resultados do presente estudo indicam a melhora dos parâmetros fisiológicos, principalmente frequência cardíaca, e sono, indicando que a fisioterapia aquática pode ser um recurso importante para proporcionar conforto e favorecer o desenvolvimento do recém-nascido.
\end{abstract}

Palavras-chave: Recém-nascido prematuro; Hidroterapia; Unidade de Terapia Intensiva Neonatal; Dor.

\begin{abstract}
Objective: To investigate the effects of aquatic physical therapy in hospitalized premature infants in the Neonatal Intensive Care Unit. Method: This is a non-randomized clinical trial. The sample consisted of 15 preterm newborns. The personal and clinical data of the preterm newborns were collected from the medical record. They were evaluated on two consecutive days: on the first day, the variables of the study (heart and respiratory rate, peripheral saturation, blood pressure, pain and sleep) were evaluated, soon after the blood puncture for the collection of routine exams, and then, the variables were reassessed. On the second day, the procedures of the first day were repeated, however, after the puncture, aquatic physiotherapy was performed. Results: It was observed that the variables heart rate $(p<0,01)$ and sleep $(\mathrm{p}<0,01)$ showed significant differences between pre- and post-intervention. The variables saturation, respiratory rate and pain showed significant differences between pre- and post-procedure of the first day, but not between preand post-intervention. However, it was possible to observe a difference between the evaluation after the first day and the second day. Conclusion: The 36 results of the present study indicate an improvement in the physiological parameters, mainly heart rate and sleep, indicating that aquatic physiotherapy may be an important resource to provide comfort and favor the development of the newborn.
\end{abstract}

Keywords: Premature newborn; Hydrotherapy; Unit of Neonatal Intensive Care; Pain.

\section{Resumen}

Objetivo: Verificar los efectos de la fisioterapia acuática en prematuros ingresados en la Unidad de Cuidados Intensivos Neonatales. Método: Este es un ensayo clínico no aleatorizado. La muestra estuvo constituida por 15 recién nacidos prematuros. Los datos personales y clínicos de los bebés prematuros se obtuvieron de los registros médicos. 
Fueron evaluados en dos días consecutivos: el primer día se evaluaron las variables de estudio (frecuencia cardíaca y respiratoria, saturación periférica, presión arterial, dolor y sueño); poco después se realizó una punción sanguínea para recolectar las pruebas de rutina y, finalmente, se reevaluaron las variables. El segundo día se repitieron los procedimientos del primer día; sin embargo, después de la punción de sangre, se realizó fisioterapia acuática. Resultados: Se pudo observar que las variables frecuencia cardíaca $(\mathrm{p}<0.01)$ y sueño $(\mathrm{p}<0.01)$ mostraron diferencias significativas entre pre y posintervención. Las variables saturación, frecuencia respiratoria y dolor mostraron diferencias significativas entre el pre y postoperatorio el primer día, pero no entre el pre y posintervención. Sin embargo, fue posible observar diferencias entre la evaluación después del primer día y después del segundo día. Conclusión: Los resultados del presente estudio indican una mejora en los parámetros fisiológicos, especialmente la frecuencia cardíaca y el sueño, indicando que la fisioterapia acuática puede ser un recurso importante para brindar comodidad y favorecer el desarrollo del recién nacido.

Palabras clave: Recién nacido prematuro; Hidroterapia; Unidad de Terapia Intensiva Neonatal; Dolor.

\section{Introdução}

O parto prematuro é definido como a ocorrência do nascimento com 37 semanas de gestação ou menos, ou seja, crianças nascidas antes da maturidade fetal. O nascimento de prematuros representa um grande desafio para os serviços de saúde pública em todo o mundo, por tratar-se de um determinante de morbidade e mortalidade neonatal (Almeida et al., 2005). A etiologia do parto prematuro é multifatorial e a preocupação constante com os recém-nascidos prematuros, bem como com as condições perinatais que sobre eles repercutem devem ser consideradas para evitar o nascimento precoce (Melo \& Carvalho, 2014).

Com o nascimento de um recém-nascido prematuro, faz-se necessário um local que possua recursos tecnológicos, humanos e terapêuticos especializados, a fim de proporcionar a ele cuidados mais complexos. Esses suportes são encontrados nas Unidades de Terapia Intensiva Neonatal (UTIN), onde a assistência ocorre de maneira contínua e cujo surgimento tem proporcionando um aumento na sobrevida orgânica dos prematuros, principalmente os extremos e de baixo peso (Santos, et al., 2012).

Na UTIN há predomínio de situações adversas; dentre elas, destacam-se o ambiente com luminosidade e temperatura artificial, o barulho incômodo e estressante e, ainda, a quantidade de manipulações realizadas nestes pacientes, em geral agressivas e dolorosas. Desta maneira, ao ser hospitalizado na UTIN, o recém-nascido prematuro fica exposto à realização de técnicas e procedimentos invasivos e potencialmente dolorosos, que poderão impactar em sua qualidade de vida e desenvolvimento neuropsicomotor (Santos, Pereira et al., 2012).

O recém-nascido prematuro possui todos os componentes funcionais e neuroquímicos necessários para a recepção e transmissão do estímulo doloroso. Nessa perspectiva, para realizar um manuseio adequado, faz-se necessário conhecer as respostas não verbais do recém-nascido prematuro referentes à dor, que geralmente compreendem reações comportamentais e autonômicas (Marcondes et al., 2017).

Para Marcondes et al., (2017), no contexto da avaliação da dor nos recém-nascidos (RNs), os principais parâmetros usados são os comportamentais e fisiológicos. Para os parâmetros fisiológicos, existem mudança na frequência cardíaca, frequência respiratória, pressão arterial e saturação de oxigênio, enquanto os comportamentais envolvem as expressões faciais, os movimentos corporais e o choro.

Estudo realizado por Nicolau et al., (2008) observou uma queda acentuada na saturação de oxigênio após estímulo doloroso em recém-nascidos prematuros. Silva et al., (2018), durante a punção arterial, avaliaram a dor em RNs e observaram que após o estímulo nociceptivo o RN apresenta aumento da frequência cardíaca (FC) e queda da saturação de oxigênio.

Somado a isso, durante a internação na UTIN o RN está exposto a um ambiente estimulante, pois o ruído é um dos perturbadores mais importantes do ritmo do sono e produz sérios efeitos na saúde mental. O RN exposto a altos níveis de ruídos pode sofrer distúrbios nos padrões de sono e apresentar irritabilidade, agitação e choro (Gaíva et al., 2010). 
O estudo de Orsi et al., (2015) relatam que a privação do sono dos recém-nascidos prematuros na unidade neonatal pode acarretar piora do estado clínico e retardar a alta hospitalar, além de desencadear distúrbios comportamentais na vida adulta. Os recém-nascidos prematuros apresentam o sono com padrão polifásico que se classifica em três estágios: sono ativo, quieto e indeterminado, sendo que os diferentes estágios de sono são fundamentais para a homeostase do organismo, pois estabelecem relação com importantes atividades fisiológicas e neurológicas (Silva et al., 2017).

Diante da complexidade do cuidado do recém-nascido prematuro em unidade neonatal, há urgente necessidade do uso de técnicas e medidas terapêuticas pautadas na assistência humanizada que possam minimizar o impacto negativo da prematuridade nos parâmetros fisiológicos de recém-nascidos prematuros. Umas das técnicas terapêuticas mais aplicadas neste sentido nas unidades neonatais brasileiras é a hidroterapia. $\mathrm{O}$ recém-nascido é imerso em água morna em uma banheira até a altura dos ombros/clavículas e é suave e passivamente mobilizado por meio de flutuação assistida (Acosta, 2010).

A fisioterapia aquática, vem sendo também utilizada para o tratamento de recém-nascidos (RN) internados em Unidade de Terapia Intensiva Neonatal (UTIN). Dentre os benefícios da fisioterapia aquática estão o desenvolvimento do sistema biológico em prematuros, promoção do ganho de peso e tolerância alimentar, redução da dor, melhora da qualidade do sono, redução dos efeitos dolorosos provocados pelos procedimentos rotineiros da UTIN e estimulação tátil necessária para o seu neurodesenvolvimento (Valizadeh et al., 2017).

O Watsu, criado em 1980, consiste em um método da fisioterapia aquática que mostrou eficiência ao tratar dores crônicas e uma grande variedade de enfermidades. Foi introduzido como uma modalidade primária da reabilitação por terapeutas aquáticos e passou a ser ensinado em universidades em todo o mundo, mostrando benefícios físicos resultantes dos movimentos e alongamentos facilitados pela utilização da água morna (Acosta, 2010).

Para Vignochi et al., (2010) relatam que a técnicas de banho podem promover tanto a redução do choro e da angústia como a melhora do comportamento e qualidade do sono. A estimulação tátil e cinestésica, por sua vez, têm mostrado efeitos benéficos na redução da dor. Assim como Barbosa et al., (2015) identificaram resultados como melhora da saturação periférica de oxigênio, diminuição da frequência respiratória e cardíaca e diminuição nos níveis de cortisol com a fisioterapia aquática em RNs internados na unidade de cuidados intermediários.

Considerando o alto número de manuseios no recém-nascido prematuro internado na UTIN, muitos deles dolorosos, é pertinente minimizar os efeitos da dor, usando técnicas não farmacológicas como a fisioterapia aquática. Embora existam algumas pesquisas, ainda se considera escassa a literatura sobre o tema. Dessa forma, o estudo justifica-se pela necessidade de ampliar a avaliação dos efeitos da fisioterapia aquática em prematuros internados na UTIN.

A partir dessa visão da fisioterapia aquática em RN prematuros internados na UTIN e na perspectiva de contribuir para a analgesia, este estudo objetivou verificar os efeitos da fisioterapia aquática após procedimentos dolorosos nos sinais vitais, dor e sono em recém-nascidos prematuros.

\section{Metodologia}

Trata-se de um ensaio clínico não randomizado, do tipo séries temporais, de abordagem quantitativa. $\mathrm{O}$ estudo foi realizado em um hospital de médio porte, localizado na Região Noroeste do Rio Grande do Sul, na Unidade de Terapia Intensiva Neonatal.

A coleta de dados ocorreu no período de março de 2018 a agosto de 2018, após autorização do responsável pelo local, aprovação pelo Comitê de Ética em Pesquisa com Seres Humanos (CEP) da Universidade Franciscana, parecer número 2.325.525 (CAAE: 77675417.9.0000.5306) e leitura e assinatura do Termo de Consentimento Livre e Esclarecido (TCLE) pelos responsáveis, formalizando o aceite. O estudo segue todas as diretrizes e normas preconizadas pela Resolução 466/12 do 
Conselho Nacional de Saúde. Ainda, o estudo foi registrado no Registro Brasileiro de Ensaios Clínicos (ReBEC), sob número U1111-1202-2302.

A amostra foi composta por RNs prematuros que atenderam aos seguintes critérios de inclusão: internados no período de março de 2018 a agosto de 2018 que estavam com quadro clínico estável, em ar ambiente ou oxigenoterapia via óculos nasal 0,5 L/m, permanência mínima na UTIN de sete dias, alimentação orogástrica e/ou oral e assinatura do TCLE pelos pais e/ou responsável. Os critérios de exclusão foram: prematuro em ventilação mecânica invasiva ou ventilação mecânica não invasiva, pré ou pós-operatório imediato, com lesões na pele, aqueles com doença neurológica, com uso de sedativos e sem prescrição de fisioterapia.

Os dados pessoais e clínicos do RN foram coletados do prontuário e transcritos para a ficha de avaliação, que também incluiu as informações sobre as variáveis do estudo, FC, frequência respiratória (FR), pressão arterial média (PAM), saturação periférica de $\mathrm{O} 2(\mathrm{SpO} 2)$, dor e sono.

Para a verificação dos sinais vitais (FC, PAM e SpO2) foi utilizado um monitor multiparâmetros Dash 4000, marca GE. Para a verificação da FR foi realizada observação do movimento abdominal durante 1 minuto. A dor foi avaliada através da escala Premature Infant Pain Profile (PIPP), que consiste em uma medida multidimensional de sete itens de dor, que tem sido amplamente utilizada para avaliar a dor aguda em bebês prematuros (Gibbins et al, 2014). Por fim, o sono foi avaliado através da escala de avaliação do estado de sono e vigília, adaptada de Brazelton, codificada de 1 à 6 sendo: 1= sono profundo, $2=$ sono leve, $3=$ sonolência, $4=$ alerta inativo, $5=$ alerta com atividade, $6=$ choro, com apenas uma alternativa possível para cada avaliação (Comaru, 2005).

Os RNs prematuros foram avaliados em dois dias consecutivos: no primeiro dia, foi realizada a verificação dos sinais vitais FC, FR, SpO2, PAM, sono e dor. Na sequência, foi realizado o procedimento doloroso (punção sanguínea) para coleta de exames conforme rotina da UTIN e após foram reavaliadas as variáveis do estudo. Toda avaliação foi realizada no período da manhã entre $7 \mathrm{~h}$ e $7 \mathrm{~h} 15 \mathrm{~min}$.

No segundo dia, foi realizado o procedimento doloroso (punção sanguínea), após verificação dos sinais vitais FC, FR, Spo2, PAM, sono e dor e, então, foi realizada a fisioterapia aquática. A intervenção teve duração de 10 minutos e foi realizada em uma banheira com água morna em torno de $37^{\circ}$, monitorada com termômetro. O RN foi imerso até os ombros, com touca para evitar a perda excessiva de calor. Foram realizado movimentos rítmicos e lentos, associados com mobilizações de cinturas e alongamentos de membros inferiores e superiores baseados no método de hidroterapia Watsu (Edraki et al., 2014). Em alguns momentos, o RN foi posicionado em padrão flexor para proporcionar relaxamento e segurança. Finalizou-se a terapia com o $\mathrm{RN}$ na incubadora ou berço aquecido posicionado em decúbito dorsal e membros fletidos junto ao corpo. Por fim, as variáveis do estudo foram reavaliadas.

\section{Quadro 1.}

\begin{tabular}{|c|c|}
\hline $1^{\circ}$ dia & $2^{\circ} \mathrm{dia}$ \\
\hline $\begin{array}{l}1^{\circ} \text { Avaliação dos sinais vitais: } \mathrm{FC}, \mathrm{FR}, \mathrm{SpO} 2, \mathrm{PAM} \text {, } \\
\text { sono e dor. } \\
2^{\circ} \text { Procedimento doloroso. } \\
3^{\circ} \text { Reavaliação: FC, FR, } \mathrm{SpO} 2 \text {, sono e dor. }\end{array}$ & $\begin{array}{l}1^{\circ} \text { Procedimento doloroso } \\
2^{\circ} \text { Avaliação dos sinais vitais: FC, FR, SpO2, } \\
\text { PAM, sono e dor. } \\
3^{\circ} \text { Fisioterapia aquática } \\
4^{\circ} \text { Reavaliação: FC, FR, SpO2, PAM, sono e } \\
\text { dor. }\end{array}$ \\
\hline
\end{tabular}

Fonte: Autores. 
A análise dos dados foi feita por meio de estatística descritiva (média, desvio-padrão e frequências) e estatística inferencial. A normalidade e a homogeneidade dos dados foram testadas pelo teste de Kolmogorov-Smirnov e Shapiro-Wilk, respectivamente. Os dados das quatro avaliações foram analisados por meio do teste de Friedman, seguido do teste post hoc de Dunn. A associação e relação entre as variáveis foram avaliadas através do teste qui-quadrado, teste $t$ de amostras dependentes e Wilcoxon. Foi usado um nível de significância de alfa igual a 5\%. O software utilizado para as análises foi o Statistical Package for the Social Sciences (SPSS), versão 23.0.

\section{Resultados}

Participaram do estudo 15 RNs prematuros. As características da amostra são apresentadas na Tabela 1. Na Tabela 2, estão descritas as variáveis nos quatro momentos de avaliação. Já a Tabela 3 apresenta os resultados do teste post hoc, que permite identificar as diferenças entre as avaliações.

A Tabela 1 expõe que 80\% da amostra foi composta pelo sexo masculino, a idade gestacional prevalece entre 35 e 37 semanas, e o peso dos prematuros varia de $500 \mathrm{~g}$ até $3000 \mathrm{~g}$, a causa maior para a internação dos recém-nascidos na UTIN é diagnóstico de prematuridade + baixo peso.

Tabela 1. Características da amostra.

\begin{tabular}{lcc}
\hline Sexo & $\mathbf{N}^{\mathbf{0}}$ & $\%$ \\
Masculino & 12 & 80 \\
Feminino & 3 & 20 \\
\hline Idade Gestacional & $\mathbf{N}^{\mathbf{0}}$ & $\%$ \\
$25-29$ Semanas & 4 & 26,66 \\
$30-34$ Semanas & 5 & 33,33 \\
$35-37$ Semanas & 6 & 40,00 \\
\hline Peso & $\mathbf{N}^{\mathbf{0}}$ & $\%$ \\
500 - 1000 g & 3 & 20 \\
1000 - 1500 g & 1 & 6,66 \\
1500 - 2000 g & 5 & 33,33 \\
2000 - 2500 g & 1 & 6,66 \\
2500 - 3000 g & 6 & 40 \\
\hline Diagnóstico de internação & $\mathbf{N}^{0}$ & $\%$ \\
Prematuridade & 2 & 13,33 \\
Prematuridade + baixo peso & 7 & 46,66 \\
Prematuridade + disfunção respiratória & 6 & 40 \\
\hline
\end{tabular}

Fonte: Dados coletados pelos autores. 
Foi possível observar na Tabela 2 que as variáveis FC e sono mostraram diferenças significativas entre pré e pósprocedimento e pré e pós-intervenção. As variáveis $\mathrm{FR}, \mathrm{SpO} 2$ e dor mostraram diferenças significativas entre pré e pósprocedimento do primeiro dia (sem intervenção), mas não entre pré e pós-intervenção (segundo dia). No entanto, foi possível observar diferença significativa entre a avaliação pós do primeiro dia, em que não houve intervenção e pós do segundo dia, no qual houve intervenção. A PAM não mostrou diferenças significativas após procedimento doloroso e após intervenção com fisioterapia aquática.

Tabela 2. Frequência respiratória (FR), frequência cardíaca (FC), saturação periférica de oxigênio (Spo2), pressão arterial média (PAM), dor e sono nos quatro momentos de avaliação $(\mathrm{n}=15)$

\begin{tabular}{ccccccccc}
\hline Variáveis & N & Média & DP & Mediana & Mínimo & Máximo & Teste estatístico & $p$ \\
\hline FR1 & 15 & 49,6 & 14,75 & 44 & 33 & 73 & Friedman & $0,002^{*}$ \\
FR2 & 15 & 57,6 & 14,69 & 57 & 30 & 85 & & \\
FR3 & 15 & 48,33 & 11,35 & 43 & 36 & 70 & & \\
FR4 & 15 & 49,47 & 10,38 & 53 & 34 & 65 & & \\
\hline FC1 & 15 & 149,67 & 13,42 & 145 & 129 & 173 & Friedman & $<0,001^{*}$ \\
FC2 & 15 & 162,8 & 16,58 & 161 & 143 & 201 & & \\
FC3 & 15 & 157,4 & 16,08 & 155 & 140 & 192 & & \\
FC4 & 15 & 148,8 & 16,34 & 145 & 132 & 196 & & \\
\hline SpO2 1 & 15 & 96,53 & 2,33 & 97 & 92 & 100 & Friedman & $<0,001^{*}$ \\
SpO2 2 & 15 & 92 & 4,77 & 94 & 83 & 98 & & \\
SpO2 3 & 15 & 95,81 & 1,82 & 96 & 93 & 98 & & \\
SpO2 4 & 15 & 96,47 & 2,75 & 97 & 88 & 100 & & \\
\hline PAM1 & 15 & 62,93 & 16,54 & 59 & 39 & 89 & Friedman & \\
PAM2 & 15 & 65,13 & 16,14 & 61 & 41 & 87 & & \\
PAM3 & 15 & 64,26 & 13,56 & 66 & 35 & 86 & & \\
PAM4 & 15 & 62,07 & 12,09 & 66 & 38 & 81 & & \\
\hline Sono1 & 15 & 1,4 & 0,63 & 1 & 1 & 3 & Friedman & $<0,001^{*}$ \\
Sono2 & 15 & 4,07 & 1,79 & 4 & 1 & 6 & & \\
Sono3 & 15 & 1,4 & 0,91 & 1 & 1 & 4 & & \\
Sono4 & 15 & 3,2 & 0,77 & 3 & 2 & 5 & & \\
\hline Dor1 & 15 & 4,4 & 1,51 & 4 & 3 & 7 & Friedman & $<0,001^{*}$ \\
Dor2 & 15 & 6,13 & 2,64 & 5 & 2 & 12 & & \\
Dor3 & 15 & 4 & 1,07 & 4 & 3 & 6 & & \\
Dor4 & 15 & 3,73 & 1,79 & 4 & 2 & 9 & & \\
\hline
\end{tabular}

$\mathrm{FR}=$ respirações por minuto; $\mathrm{FC}=$ batimentos por minuto; $\mathrm{SpO} 2=\% ; \mathrm{PAM}=\mathrm{mmHg}$ $* \mathrm{p} \leq 0,05$

Fonte: Dados coletados pelos autores.

Na Tabela 3 não foi observada associação significativa entre sono e dor $(\mathrm{p} \geq 0,05)$ em nenhum dos quatro momentos de avaliação. Também não houve relação entre sono e os sinais vitais e dor e sinais vitais $(p \geq 0,05)$ em nenhuma das avaliações, exceto para sono e FC na avaliação pós-procedimento doloroso no primeiro dia $(\mathrm{p}<0,01)$. 
Tabela 3. Post hoc para identificação de diferença entre os quatro momentos de avaliação.

\begin{tabular}{|c|c|c|c|c|}
\hline FR & FR1 & FR2 & FR3 & FR4 \\
\hline FR1 & $\mathrm{X}$ & $0,014^{*}$ & 1 & 1 \\
\hline FR2 & $\mathrm{x}$ & $\mathrm{x}$ & 0,08 & $0,002^{*}$ \\
\hline FR3 & $\mathrm{x}$ & $\mathrm{x}$ & $\mathrm{x}$ & 1 \\
\hline FR4 & $\mathrm{x}$ & $\mathrm{x}$ & $\mathrm{x}$ & $\mathrm{x}$ \\
\hline $\mathrm{FC}$ & FC1 & $\mathrm{FC} 2$ & FC3 & $\mathrm{FC} 4$ \\
\hline $\mathrm{FC} 1$ & $\mathrm{x}$ & $0,001^{*}$ & 0,065 & 1 \\
\hline $\mathrm{FC} 2$ & $\mathrm{x}$ & $\mathrm{x}$ & 1 & $<0,001^{*}$ \\
\hline FC3 & $\mathrm{x}$ & $\mathrm{x}$ & $\mathrm{x}$ & $0,002^{*}$ \\
\hline $\mathrm{FC} 4$ & $\mathrm{x}$ & $\mathrm{x}$ & $\mathrm{x}$ & $\mathrm{x}$ \\
\hline $\mathrm{SpO} 2$ & $\mathrm{SpO} 21$ & $\mathrm{SpO} 22$ & $\mathrm{SpO} 23$ & $\mathrm{SpO} 24$ \\
\hline SpO2 1 & $\mathrm{x}$ & $0,007^{*}$ & 1 & 1 \\
\hline $\mathrm{SpO} 22$ & $\mathrm{x}$ & $\mathrm{x}$ & $0,035^{*}$ & $<0,001^{*}$ \\
\hline SpO2 3 & $\mathrm{x}$ & $\mathrm{x}$ & $\mathrm{x}$ & 0,944 \\
\hline $\mathrm{SpO} 24$ & $\mathrm{x}$ & $\mathrm{x}$ & $\mathrm{X}$ & $\mathrm{x}$ \\
\hline SONO & SONO1 & SONO2 & SONO3 & SONO4 \\
\hline SONO1 & $\mathrm{x}$ & $0,002^{*}$ & 1 & $0,018^{*}$ \\
\hline SONO2 & $\mathrm{x}$ & $\mathrm{x}$ & $0,001 *$ & 1 \\
\hline SONO3 & $\mathrm{x}$ & $\mathrm{x}$ & $\mathrm{x}$ & $0,014^{*}$ \\
\hline SONO4 & $\mathrm{x}$ & $\mathrm{x}$ & $\mathrm{x}$ & $\mathrm{x}$ \\
\hline DOR & DOR1 & DOR2 & DOR3 & DOR4 \\
\hline DOR1 & $\mathrm{x}$ & 0,286 & 1 & 0,538 \\
\hline DOR2 & $\mathrm{x}$ & $\mathrm{x}$ & $0,028^{*}$ & $0,001^{*}$ \\
\hline DOR3 & $\mathrm{x}$ & $\mathrm{x}$ & $\mathrm{x}$ & 1 \\
\hline DOR4 & $\mathrm{x}$ & $\mathrm{x}$ & $\mathrm{x}$ & $\mathrm{x}$ \\
\hline
\end{tabular}

$1=$ variáveis coletas antes do procedimento doloroso; 2 = variáveis coletas após procedimento doloroso; $3=$ variáveis coletas após procedimento doloroso e antes da fisioterapia aquática; 4= variáveis coletadas após a fisioterapia aquática * $\mathrm{p} \leq 0,05$

Fonte: Dados coletados pelos autores.

\section{Discussões}

O presente estudo visou conhecer os efeitos da fisioterapia aquática em RN prematuros internados na UTIN. De acordo com os resultados, a fisioterapia aquática tem um efeito positivo principalmente nas variáveis FC e sono, mas também pode contribuir para estabilizar a FR, a SpO2 e a dor.

Os RNs prematuros, após receberem algum tipo de intervenção dolorosa como, por exemplo, punção sanguínea, demonstraram taquicardia, agitação e choro intenso. Após serem submetidos à fisioterapia aquática, os batimentos cardíacos diminuíram, estabilizando o $\mathrm{RN}$ e proporcionando tranquilidade em menor tempo, como se não tivessem passado pelo procedimento de punção sanguínea. Observou-se diferença significativa entre pós-procedimento doloroso e pós-intervenção com fisioterapia aquática (primeiro e segundo dia).

Alguns estudos relataram que a hidroterapia promove relaxamento muscular com consequente redução dos parâmetros clínicos como a FC. Estes achados são semelhantes no presente estudo, pois os RNs submetidos à fisioterapia aquática 
apresentaram diminuição na FC. Para Barbosa et al., (2015) relatam diminuição na média da FC em 10 recém-nascidos hospitalizados submetidos à hidroterapia por um período de 10 minutos, assim como para Vignochi et al., (2010). Antes do procedimento de terapia aquática em 12 RNPT, a média da FC era de 172 batimentos por minuto; cinco minutos após a intervenção, a média diminuiu para 157 batimentos por minuto; no decorrer de 30 minutos, a média reduziu para 147 batimentos por minuto.

O sono dos RNs prematuros após procedimento doloroso sofreu alterações, passando de estado 1 sono profundo, segundo a escala adaptada de Brazelton, para estado 6 choroso; esse estado favorece a instabilidade hemodinâmica do RN. Após 10 minutos de terapia aquática, o RN passou para um estado 3 que significa sonolento, retornando para um estado comportamental favorável para o seu neurodesenvolvimento. Esses resultados condizem com o estudo de Vignochi et al., (2010), em que 12 recém-nascidos pré-termo e internados na UTIN apresentaram comportamento que variaram entre totalmente acordado, movimentos vigorosos e choroso e, após, a fisioterapia aquática os estados variaram entre sono leve e algum movimento corporal.

A FR aumentou pós-procedimento doloroso no primeiro dia sem intervenção e não apresentou diferenças no segundo dia antes e após a intervenção. Entretanto, houve diferença entre pós-procedimento doloroso (primeiro dia) e pós-intervenção segundo dia. Já a SpO2 diminuiu no pós-procedimento doloroso no primeiro dia sem intervenção e não apresentou diferenças no segundo dia antes e após a intervenção. No entanto, também apresentou diferença entre pós-procedimento doloroso e pósintervenção. Após o procedimento doloroso os RNs, encontravam-se agitados e chorosos, o que causa dispneia e queda da saturação periférica de oxigênio. Após a fisioterapia aquática, houve melhora no estado comportamental e consequente melhora dos parâmetros de FR e SpO2.

Para Tobinaga et al., (2016) a FR pré foi de 55,2 respirações por minuto, após a intervenção com a hidrocinesioterapia houve redução para 49,2 respirações por minuto. A SpO2 foi de 97\% antes da intervenção e após passou para 99\%. Vignochi et al., (2010) observou no seu estudo que a FR apresentou redução significativa após a intervenção aquática, principalmente após os 60 minutos do término do procedimento. Antes da fisioterapia aquática, os bebês encontravam-se agitados e chorosos, apresentando uma média de $\mathrm{SpO} 2$ de $91 \%$. Após a fisioterapia aquática, houve aumento estatisticamente significativo na SpO2, sobretudo após 60 minutos da intervenção, para 97\%.

No presente estudo, na avaliação da dor pela escala PIPP não foi observada diferença estatística pós-intervenção. Porém, houve diferença significativa entre o pós-procedimento doloroso no primeiro dia e o pós-intervenção no segundo dia. Isso significa que a fisioterapia aquática foi capaz de estabilizar a dor do RN.

Também Tobinaga et al., (2016) demonstraram uma redução significativa nos níveis de cortisol salivar após a hidrocinesioterapia, indicando que a terapia pode reduzir a dor, o estresse, a irritabilidade e as alterações adquiridas no RN durante longos períodos de hospitalização. Barbosa et al., (2015) também relataram a redução significativa da dor póshidroterapia, avaliada por meio da escala NIPS.

No estudo de Vignochi et al., (2010) a avaliação da dor por meio do Sistema de Codificação da Atividade Facial Neonatal mostrou uma redução significativa após a fisioterapia aquática. Os efeitos fisiológicos da água produzem alívio da dor e relaxamento muscular geral por meio da redução da sensibilidade dos terminais nervosos e do aumento da circulação periférica, o qual provê maior suprimento de sangue para os músculos.

Diante da dor e estresse sofrido pelo recém-nascido prematuro é importante a necessidade de incluir técnicas como a fisioterapia aquática que possam oferecer maior qualidade de vida e gerar homeostase para esse prematuro Brito et al., (2020).

$\mathrm{Na}$ literatura, foram encontrados poucos estudos que avaliassem a variável PAM, o que torna difícil a comparação. Assim como, Vignochi et al., (2010) avaliaram a PAM após a fisioterapia aquática e, assim como no presente estudo, não houve alteração significativa antes e após o procedimento. 
Uma hipótese para a ausência de efeitos na PAM é que, mesmo alterando os sinais vitais, o procedimento doloroso e a intervenção podem não ter promovido mudanças substanciais no débito cardíaco e resistência vascular sistêmica capazes de modificar a pressão arterial.

Conforme os resultados deste estudo, os RNs apresentavam-se taquicárdicos após o procedimento doloroso, provavelmente em função do comportamento choroso e ou da presença de dor. Após a fisioterapia aquática, a FC reduziu, sugerindo que esteja associada à mudança comportamental de choroso para sonolento após o procedimento, como nos estudos de Vignochi et al., (2010) e Barbosa et al., (2015).

Mesmo não apresentando efeitos em todas as variáveis analisadas, a fisioterapia aquática aplicada no presente estudo mostrou benefícios que podem colaborar para o desenvolvimento e recuperação do RN prematuro, aliviando sintomas e promovendo melhora na qualidade de vida.

Estudo realizado por Alves et al., (2020) afirmam ao avaliar parâmetros vitais (FC, FR e SpO2) após cada banho de higiene, percebeu-se valores mais estáveis em todos os parâmetros avaliados nas crianças que realizaram ofurô.

E nesse ambiente hospitalar é oportuno repensar em ações que visam a humanização. Humanizar não é uma técnica, mas sim um processo vivencial que permeia todas as atividades das pessoas que assistem o paciente, procurando realizar e oferecer o tratamento que ele merece (Reichert et al., 2007).

\section{Conclusão}

A fisioterapia aquática mostrou-se efetiva e segura para RNs prematuros internados na UTIN, principalmente no que se refere à FC e sono. Já para a FR, SpO2 e dor a intervenção promoveu resultados positivos, especialmente quando se comparou os resultados do pós-procedimento doloroso no primeiro dia com os do pós-intervenção no segundo dia, indicando estabilização das variáveis. A PAM foi única variável que não apresentou alterações importantes. Assim, a fisioterapia aquática pode ser indicada para RNs prematuros como um recurso não farmacológico que promove alívio, relaxamento e conforto, contribuindo para a redução dos efeitos nocivos do tratamento intensivo dentro de uma UTIN e favorecendo a recuperação da saúde e o desenvolvimento do RN.

O presente estudo apresentou limitações que devem ser apontadas, pois o grupo analisado ainda é pequeno, havendo dificuldade de generalização de resultados, sendo a principal limitação do estudo a ausência de um grupo controle, assim como mudanças nas condições ambientais, efeito do horário das avaliações e a rotina da UTIN. Com base nas discussões levantadas e nas limitações deste estudo, sugere-se a condução de estudos controlados e com maior número amostral, diferentes variáveis e maior período de intervenção para examinar a presença de possíveis fatores de confusão que poderiam interferir nos resultados.

\section{Referências}

Acosta, A. M. C. (2010) Comparação das técnicas Watsu e relaxamento aquático em flutuação assistida nos sintomas de ansiedade, depressão e percepção da dor. Dissertação. Programa de Pós-Graduação em Psicologia da Saúde, Universidade Metodista de São Paulo. Brasil.

Almeida, A. C., Jesus, A. C. P., Lima, P. F. T., Araújo, M. F. M., \& Araújo, T. M. (2012) Fatores de risco maternos para prematuridade em uma maternidade pública de Imperatriz - MA. Rev Gaúcha Enferm. 55(2), 86-94. https://doi.org/10.1590/S1983-14472012000200013

Alves, M. V., Cantalice, A. da S. C., Azevedo, E. B. de, Silva, H. M. F. de A., \& Barbosa, J. C. G. (2020). Comparação do ofurô com o banho de higiene após procedimentos invasivos. Research, Society and Development, 9(10), e7189108882. https://doi.org/10.33448/rsd-v9i10.8882

Barbosa, L. P. C., Carneiro, E. M., \& Weffort, V. (2015) Impacto Da Hidroterapia Em Recém-Nascidos Hospitalizados. Fisiot Bras. 16(3), 207-211. https://Doi.Org/10.33233/Fb.V16i3.76

Batten, M., Stevenson, E., Zimmermann, D., \& Isaacs, C. (2017) Implementation of a hydrotherapy protocol to improve postpartum pain management. $J$. Midwifery Womens Health. 62 (2), 210-214. 
Brito, R.S., Costa, C. L. A., Reis, L.J., Cruz, C. B., \& Sá, L. t. S., Paciência, J. S.(2020). A utilização da ofurôterapia para recém-nascidos pré-term hospitalizados. Revista Eletrônica Acervo Saúde,40, 1- 7 https:// doi.org/1025248/reas.e27342020

Comaru, T.(2005) Efeitos fisiológicos e comportamentais de um protocolo de contenção postural em recém-nascidos pré-termo. (2005) Dissertação. Universidade do Rio Grande do Sul, Faculdade de Medicina Programa de Pós-Graduação em Ciências Médicas: Pediatria. RS.Brasil

Edraki, M., Montaseri, S., Razavi, N. M., \& Montasari, Z. (2014) Comparing the effects of swaddled and conventional bathing methods on body temperature and crying duration in premature infants: a randomized clinical trial. J. Caring Sci. 1,3(2), 83-91. 10.5681/jcs.2014.009.

Gaíva, M. A. M., Marquesi, M. C., \& Rosa, M. K. O. (2010) O sono do recém-nascido internado em unidade de terapia intensiva: cuidados de enfermagem. Cienc Cuid saúde. 9(3), 602-609. https://doi.org/10.4025/cienccuidsaude.v9i3.12561

Gibbins, S., Stevens, B.J., Yamada, J., Dionne, K., Campbell-Yeo, M., Lee, G., Caddell, K., Johnston, C., \& Taddio, A.(2014) Validation of the Premature Infant Pain Profile- Revised (PIPP-R). Early Hum Dev. 90 (4), 189-193. 10.1016/j.earlhumdev.2014.01.005.

Marcondes, C., Costa, A. M. D., Chagas, E. K., Coelho, J. B. A. (2017) Conhecimento da equipe de enfermagem sobre a dor no recém-nascido prematuro. Rev enferm UFPE.11(9), 354-359. 10.5205/reuol.11088-99027-5-ED.1109201705

Melo, W. A. \& Carvalho, M. D. B. (2014) Análise multivariada dos fatores de risco para prematuridade no sul do Brasil. Rev Eletr. Gestão e Saúde. 5(2), 398-409.

Nicolau, C. M., Modesto, K., Nunes, P., Araújo, K., Amaral, H., \& Falcão, M. C. (2008) Avaliação da dor no recém-nascido prematuro: parâmetros fisiológicos versus comportamentais. Arq Bras Cienc Saúde. 33(3), 146-150.

Orsi, K. C. S. C., Llaguno, N. S., Avelar, A. F. M., Tsunemi, M. H., Pedreira, M. L. G., Sato, M. H., \& Pinheiro, E. M. (2015) Efeito da redução de estímulos sensoriais e ambientas no sono e recém-nascido pré-termo hospitalizados. Rev Esc Enferm USP. 49(4), 550-555. 10.1590/S0080-623420150000400003

Reichert, A. P. S., Lins, R. N. P., \& Collet, N.(2007) Humanização Do Cuidado Da Uti Neonatal. Rev. Eletr. Enferm. 9 (1), 200-213. Https://Doi.Org/10.5216/Ree.V9i1.7148

Santos, L. M., Pereira, M. P., Santos, L. F. N., \& Santana, R. C. B. (2012) Avaliação da dor no recém-nascido prematuro em Unidade de Terapia Intensiva. Rev Bras Enferm. 65(1), 27-33.

Santos, L. M., Ribeiro, I. S., \& Santana, R. C. B. (2012) Identificação e tratamento da dor no recém-nascido prematuro na Unidade de Terapia Intensiva. Rev Bras Enferm. 65(2), 269-275.

Silva, H. A., Silva, K. C., Reco, M. O. N., Costa, A. S., Marangoni, D. A. S., \& Merey, L. S. F. (2017) Efeitos fisiológicos da hidroterapia em balde em recémnascidos prematuros. Rev Ter Ocup Univ São Paulo 28(3), 309-315. http://dx.doi.org/10.11606/issn.2238-6149.v28i3p309-315

Silva, P. L. N., Barbosa, S. L., Rocha, R. G., Ferreira, T. N. (2018) Experience and needs of parents from premature neonates hospitalized in a neonatal intensive care unit. Rev Enferm UFPI. 7(1), 15-19.

Tobinaga, W. C. O., Marinho, C. L, Abelenda, V. L. B., Sá, P. M., \& Lopes, A. J. (2016) Short-term effects of hidrokinesiotherapy in hospitalized preterm newborns. Reabilitation on Research and Pratice. 1-8. doi.org/10.1155/2016/9285056

Valizadeh, L., Sanaeefar, M., Hosseini, M. B., Jafarabadi, M. A., \& Shamili, A.(2017) Effect of early physical activity programs on motor performance and neuromuscular development in infants born preterm: a randomized clinical trial. J. Caring Sci. 6, 67-79. 10.15171/jcs.2017.008

Vignochi, C., Teixeira, P. P., \& Nader, S. S.(2010) Efeito da fisioterapia aquática na dor e no estado de sono e vigília de recém-nascidos pré- termo estáveis internados em unidade de terapia in tensiva neonatal. Rev Bras Fisioter. 14(3), 214-220. doi.org/10.1590/S1413-35552010000300013 